\title{
Causality in Local Quantum Field Theory: Some General Results
}

\section{Iagolnitzer}

Service de Physique Théorique de Saclay^, F-91191 Gif-sur-Yvette Cedex, France

Received December 5, 1990; in revised form June 27, 1991

\begin{abstract}
General asymptotic causality properties of chronological $N$-point functions and, in massive theories, of $\mathrm{N}$-particle collision amplitudes, are derived from locality and the spectral condition. Results include specified rates of exponential fall-off, with simple and direct physical content, for large non-causal separations of points or particles in Minkowski space-time depending on values of the energy-momenta and on the mass spectrum. Relevant mathematical results on rates of exponential fall-off of generalized Fourier transforms outside their microsupports are given.
\end{abstract}

\section{Introduction}

1.1 Preliminaries. The purpose of this paper is the derivation in field theory, from locality and the spectral condition, of general asymptotic causality (also called macrocausality, properties of chronological $N$-point functions and in turn, in massive theories, of $N$-particle collision amplitudes, with specified rates of exponential fall-off in non-causal situations. Results provide in particular a general and precise expression of the idea that energy-momentum can only propagate in future causal cones, in an asymptotic sense and up to specified exponential fall-off, with some further conditions (depending on the mass spectrum) in massive theories. They generalize, complement and unify in various respects earlier works on the subject ([1-7] and references therein) as outlined below. Further results and conjectures in massive theories, on macrocausal properties in terms of real on-shell intermediate particles, linked to asymptotic completeness, will be presented elsewhere. Results of this paper are general ones that depend only on the (possibly weakened) locality condition and on the mass spectrum. (Rates of exponential fall-off in more refined properties depend also on further aspects of the models considered: unstable particles,...) The analysis and results are in particular very close to the work of

* Laboratoire de la Direction des Sciences de la Matière du Commissariat à l'Energie Atomique 
Bros-Epstein-Glaser [5], and more precisely both to Sect. 2 of [5], in which results on $N$-point functions are given in the sense of rapid fall-off (in the line of original results of [3] on collision amplitudes in particular situations), and to Sect. 3 of [5]. In the latter, local momentum-space decompositions ${ }^{1}$ into sums of boundary values of analytic functions, whose analyticity domains (in massive theories) intersect the complex mass-shell ${ }^{2}$, have been established. They might be used, via mathematical results $[8,9]$, to obtain in turn causality properties in the sense of exponential fall-off. Our methods, which develop those outlined in [6] in terms of ("microlocal," analytic) essential support or "microsupport" properties ${ }^{3}$, are more direct and efficient to that purpose, both for the treatment of $N$-point functions and in turn of $N$-particle collision amplitudes. Results on $N$-point functions also allow one to reobtain local momentum-space analyticity properties in a simpler way, through decomposition theorems ${ }^{4}$ of $[8,9]$ : see Appendix 2. As well appear, our results generalize on the other hand earlier results on rates of exponential fall-off of $N$-point functions [1,2] or collisions amplitudes [4, 7] obtained (by different methods) in particular situations: see details on $[1,2]$ in Sect. 1.2 below and on $[4,7]$ in Appendix 3.

1.2 Contents. For definiteness, we consider as in [5] the Wightman axiomatic frame work but a similar analysis can probably be carried out in the related Haag-Araki theory of local observables. On the other hand, we consider for simplicity a theory with only one basic (interacting) field, a scalar field $A(x \rightarrow A(x))$. Locality takes the form $[A(x), A(y)]=0$ if $x-y$ is space-like. A weakened formulation of locality involving only exponential fall-off in space-like directions is also sufficient for most purposes below. The spectral condition asserts in general that the spectrum of the energy-momentum operator is contained in the closed cone $V_{+}=\left\{p, p^{2} \geqq 0, p_{0} \geqq 0\right\}$, where $p^{2} \equiv p_{0}^{2}-\vec{p}^{2}$ and $p_{0}, \vec{p}$ are the energy and momentum components of $p$. We shall also consider spectral conditions with mass gap, which will as a matter of fact characterize "massive theories," and for simplicity will restrict our attention in this case to a spectrum composed of the origin, an hyperboloid $H_{+}(\mu)$ of mass $\mu>0\left(H_{+}(\mu)=\left\{p ; p^{2}=\mu^{2}, p_{0}>0\right\}\right)$ and the continuum $V_{+}(2 \mu)=\left\{p ; p^{2} \geqq 4 \mu^{2}, p_{0}>0\right\}$.

Results on $N$-point functions and $N$-particle collision amplitudes are presented in Sects. 3 and 4 respectively. An introduction and more details on contents are

\footnotetext{
${ }^{1}$ Similar decompositions follow from the results of Sect. 2 of [5] but with undesirable $C^{\infty}$ backgrounds. The method used in Sect. 3 of [5], in momentum-space, is different and is based on a generalized edge-of-the-wedge theorem of $[8,9]$

${ }^{2}$ This is not the case for the primitive analyticity domain of the $N$-point function, nor even in general (at $N>4$ ), for its holomorphy envelope, so that several terms are needed in general in these decompositions

3 The definition [8,9] of the ("microlocal," analytic) essential support is recalled in App. 1.1. It coincides [10] with the analytic wave front set and with the singular spectrum introduced independently by different methods, in [11] and [12] respectively. This common notion is also called microsupport, following a mathematical teminology proposed by $\mathbf{M}$. Sato (which has, however, no link with microlocality in field theory)

${ }^{4}$ These theorems are used in some sense in Sect. 3 of [5], but in an indirect way, the generalized edge-of-the-wedge theorem used there (see footnote 1)) being established in $[8,9]$ as a corollary of the latter
} 
given below. Some mathematical results, which complete those of $[8,9]$ and are directly useful in Sect. 3, are first presented in Sect. 2.

The simplest result on exponential fall-off properties of $N$-point functions (in Minkowski space-time) is the exponential fall-off $[1,2]$, in a massive theory, of connected Wightman $N$-point functions $W\left(x_{1}, \ldots, x_{N}\right)$ for large space-like separations of subgroups of points. In order to get more general asymptotic causality properties, in which past and future are distinguished, one has (i) to consider chronological functions $T\left(x_{1}, \ldots, x_{N}\right)$ (= vacuum expectation values of time-ordered products of field operators $A\left(x_{1}\right), \ldots, A\left(x_{N}\right)$, whose amputated Fourier transforms $\tilde{T}^{\mathrm{amp}}\left(p_{1}, \ldots, p_{N}\right)$, in a massive theory, give the $S$ matrix by restriction to the mass-shell) and (ii) to introduce localization properties in energy-momentum space. To that purpose, one may consider the action of $T$ (which is in fact a tempered distribution), or $T^{\mathrm{amp}}$, on suitable test functions $\varphi_{i, \tau}, i=1, \ldots, N$, with (asymptotic) localization properties both in space-time and (after Fourier transformation) in energy-momentum space. The simplest choice (Sect. 2 of [5], in the line of [3]) is that of test functions with Fourier transforms $\tilde{\varphi}_{i, \tau}\left(p_{i}^{\prime}\right)=\chi_{i}\left(p_{i}^{\prime}\right) \exp -i\left(p_{i}^{\prime} \cdot u_{i}\right) \tau$, where each $u_{i}$ is a given point in space-time, $\tau$ is a scalar parameter that will tend to infinity, and each $\chi_{i}$ is a $C^{\infty}$ function with compact support around a given point $p_{i} ; \varphi_{i, \tau}$ is then well localized asymptotically around the point $\tau u_{i}$ in spacetime, in the sense of rapid fall-off. Correspondingly, rapid fall-off properties of $T\left(\left\{\varphi_{i, \tau}\right\}\right)$ in the $\tau \rightarrow \infty$ limit can be derived in non-causal situations, depending on the set of points $u_{1}, \ldots, u_{N}$ and on the supports of the functions $\chi_{i}$ (as also, in a massive theory, on the mass $\mu$ ). However, it is then not possible to see actual exponential fall-off effects, and better results cannot be achieved with functions $\tilde{\varphi}_{i, \tau}$ of the form above whether $\chi_{i}$ has a compact support (because of its $C^{\infty}$ singularities at least on the boundary of its support) or not: if e.g. $\chi_{i}$ is a gaussian centered at $p_{i}$, other points $p_{i}^{\prime}$ at possibly large but fixed distances of $p_{i}$ will spoil the causality properties expected for the given points $p_{i}$. In the line of related considerations in [4] and $[13,8,9], \chi_{i}$ is then replaced by gaussian-type functions $\chi_{i, \tau}$ with width shrinking to zero as $\tau \rightarrow \infty$. More details and results on these test functions are given in Sect. 3.1, in which basic properties [5] derived from locality and spectrum on chronological (and related) functions are also recalled. Main results on exponential fall-off properties of $T\left(\left\{\varphi_{i, \tau}\right\}\right)$, or $T^{\mathrm{amp}}\left(\left\{\varphi_{i, \tau}\right\}\right)$, in non-causal situations, depending on the set of points $u_{1}, \ldots, u_{N}$ and $p_{1}, \ldots, p_{N}$ (and, in a massive theory, on $\mu$ ) are presented in Sect. 3.2, where physical comments and some complements are also given. Results relative to $T$ apply either in general or in massive theories, with various improvements in the latter case. Finally, a space-time cluster property analogous ${ }^{5}$ to that of $[1,2]$, namely the exponential fall-off, in a massive theory, of $T\left(x_{1}, \ldots, x_{N}\right)$ itself for large space-like separations is obtained in Sect. 3.3 as a byproduct.

In Sect. 4, it is explained how asymptotic causality properties, with also specified exponential fall-off properties, can be directly established in turn for $N$-particle collision amplitudes between initial and final on-shell wave functions chosen, in the line of $[4,13]$, to be mass-shell restrictions $\hat{\varphi}_{i, \tau}$ ot previous functions $\tilde{\varphi}_{i, \tau}$.

\footnotetext{
${ }^{5}$ Results on $W$ and $T$ are very close for space-like separations. As mentioned above, chronological functions are those to be considered for more general results
} 
Relevant space-time wave functions, Fourier transforms of $\hat{\varphi}_{i, \tau}\left(p^{\prime}\right) \delta_{+}\left(p^{\prime 2}-\mu^{2}\right)$ are now well localized along the space-time trajectories $\left(\tau u_{i}, p_{i}\right)$ passing through $\tau u_{i}$ and parallel to $p_{i}$. Results on rates of exponential fall-off of these functions, away from these trajectories, are established in Sect. 4.1, where the general idea of the method used for the analysis of collision amplitudes is also presented. Main results and complements are then given in Sect. 4.2.

Complementary mathematical results are given in Appendix 1. General results that complement those of $[8,9]$ on rates of exponential fall-off of generalized Fourier transforms of tempered distributions outside their microsupports are presented in Appendix 1.1: results of Sect. 2 to 4 will appear in this context as particular cases of interest in which more precise results can be established. A convolution theorem directly useful in Sect. 4.2 is then given in Appendix 1.2.

As already mentioned, Appendix 2 explains how local analyticity properties can be derived from results of Sect. 3. Finally, results of Sect. 4 are illustrated in Appendix 3 in a simple situation analogous to that of [4].

\section{Mathematical Results}

We consider a tempered distribution $f$ defined in $\mathbb{R}^{n}$, its Fourier transform $\tilde{f}$ and the generalized Fourier transform $F$ of $\tilde{f}$ defined for each $\gamma>0$ by the formula (see $[8,9]$ and references therein)

$$
F(x, p ; \gamma)=\int \tilde{f}\left(p^{\prime}\right) e^{-i p^{\prime} \cdot x} e^{-\gamma|x|\left|p^{\prime}-p\right|^{2}} d p^{\prime}
$$

In the $\gamma \rightarrow 0$ limit, $F$ reduces to $f(x)$. It is a well defined function, depending on $p$, at $\gamma>0$. The consideration of various values of $\gamma>0$ is useful for mathematical reasons and also in the application. (The values of $\gamma$ that will allow the best expression of causality will depend on the situation considered.)

Part (i) of Theorem 1 and Theorems 3,4 are the main results needed in Sect. 3. Part ( $\left.i^{\prime}\right)$ of Theorem 1 and Theorem 2 will be useful in Sect. 3.3. Part (ii) of Theorem 1 is useful if one starts from weakened formulations of locality.

In Theorem $1, C$ is a cone with apex at the origin, $C_{a}$ is the set of points $x$ whose distance $d(x)$ to the complement of $C$ is $\geqq a, d_{a}(x)$ is the corresponding distance to the complement of $C_{a}$, and $\hat{x}=x /|x|$ is the unit vector in the direction of $x$. Parts (i) and (ii) of Theorem 1 assert essentially that, if $f(x)=0$ in $C$ or if $f$ decays exponentially like $e^{-\alpha(\hat{x})|x|}$ in the directions of $C$, then, for any point $p$ and each $\gamma>0, F(x, p ; \gamma)$ decays exponentially in each direction of $C$ at least like $\exp \left\{-\frac{d(\hat{x})^{2}}{4 \gamma}|x|\right\}$ and $\exp \{-\alpha(\hat{x}, \gamma)|x|\}$ respectively, with $\alpha(\hat{x}, \gamma)$ defined in Eqs. (4), (5), up to minor changes if $f$ is a general tempered distribution. The rates of fall-off, $d(\hat{x})^{2} / 4 \gamma$ and $\alpha(\hat{x}, \gamma)$, are arbitrarily large, or arbitrarily close to $\alpha(\hat{x})$ respectively, if $\gamma$ is sufficiently small, in agreement with the relation $\lim _{\gamma \rightarrow 0} F(x, p ; \gamma)=f(x)$.

Part (i)' will be applied in particular to regularized distributions, namely to functions obtained from a distribution vanishing in $C$ by convolution with a $C^{\infty}$ function with compact support around the origin. 


\section{Theorem 1.}

(i) If $f(x)=0$ in the cone $C$, then, given any $\varepsilon>0, F(x, p ; \gamma)$ satisfies, for $x$ in $C_{\varepsilon}$, bounds of the form

$$
|F(x, p ; \gamma)|<\left[c_{\varepsilon}(\gamma|x|)^{-v / 2} \mathscr{P}(|x|,|p|, \sqrt{\gamma|x|})\right] \exp \left\{-\frac{d_{\varepsilon}(x)^{2}}{4 \gamma|x|}\right\},
$$

where $\mathscr{P}$ is a polynomial and $v$ is an integer $\left(d_{\varepsilon}(x)=\left(d(\hat{x})-\frac{\varepsilon}{|x|}\right)|x|\right)$.

(Iff is a continuous function, $\varepsilon$ can be fixed at zero and the bounds (2) reduce to $|F|<\operatorname{cst} \exp \left\{-\frac{d(\hat{x})^{2}}{4 \gamma}|x|\right\}$ if moreover $\left.\int|f(x)| d x<\infty\right)$.

(i)' If $f(x)=0$ in $C_{a}, a \geqq 0$ and if $f$ is moreover $C^{\infty}$ (with possible, at most polynomial, increase), bounds of the form (2) hold in $C_{a}$ with $\varepsilon=a, \mathscr{P}$ independent of $|p|$ and a further factor $(1+|p|)^{-v^{\prime}}$, where $v^{\prime}$ is any $\geqq 0$ integer:

$$
|F(x, p ; \gamma)|<\frac{c_{v^{\prime}}}{(1+|p|)^{v^{\prime}}}\left[(\gamma|x|)^{-v / 2} \mathscr{P}(|x|, \sqrt{\gamma|x|})\right] \exp \left\{-\frac{d_{a}(x)^{2}}{4 \gamma|x|}\right\} .
$$

(ii) If $f$ decays exponentially like $e^{-\alpha(\hat{x})|x|}$ in each direction $\hat{x}$ of $C, \alpha(\hat{x})>0$, results analogous to (i),(i)' hold with a rate of exponential fall-off in $|x|$ at least equal (or arbitrarily close $)$ to $\alpha(\hat{x}, \gamma)$, where

$$
\begin{gathered}
\alpha(\hat{x}, \gamma)=\operatorname{Sup}_{d^{\prime}(\hat{x}) \leqq d(\hat{x})} \operatorname{Inf}\left[\frac{d^{\prime}(\hat{x})^{2}}{4 \gamma}, \alpha^{\prime}(\hat{x})\right], \\
\alpha^{\prime}(\hat{x})=\operatorname{Inf}_{\hat{y},|\hat{y}-\hat{x}| \leqq d^{\prime}(\hat{x})} \alpha(\hat{y})\left(1-d^{\prime}(\hat{x})\right) .
\end{gathered}
$$

Proof (Outline). We present below a direct proof based on the (easily checked) convolution formula:

$$
(\gamma|x|)^{n / 2} F(x, p ; \gamma)=\int f\left(x^{\prime}\right) e^{i p \cdot\left(x-x^{\prime}\right)} e^{-\left|x-x^{\prime}\right|^{2} /(4 \gamma|x|)} d x^{\prime}
$$

The integration domain is divided into the region $\left|x-x^{\prime}\right|<d(x)$ or $\left|x-x^{\prime}\right|<d^{\prime}(x)$, in which the conditions on $f$ are used, and its complement, up to slight modifications (in usual ways) if $f$ is not a continuous function but a (tempered) distribution. The further factor $(1+|p|)^{-v^{\prime}}$ in (i)' is obtained by usual methods in view of the presence of the factor $e^{-i p \cdot x^{\prime}}$. Q.E.D.

Theorem 2 is a converse of Theorem 1. It applies in cases when $f$ is e.g. a continuous function satisfying $($ at $x \neq 0)$

$$
f(x)=(\gamma|x|)^{n / 2} \int F(x, p ; \gamma) d p
$$

for some $\gamma>0$. This is the case $(\forall \gamma>0)$ if $f$ is sufficiently regular, i.e. equivalently if $\tilde{f}$ has a sufficient decrease as $|p| \rightarrow \infty$.

Theorem 2. If $f$ is a (continuous) function satisfying Eq. (7) for some fixed $\gamma_{0}>0$ and if $F$ satisfies moreover, for $x$ in $C,|x|$ larger than a given constant and $v_{0}$ sufficiently large, bounds of the form

$$
|F(x, p ; \gamma)|<C_{v_{0}}(1+|p|)^{-v_{0}} \mathscr{P}(|x|) e^{-\alpha(\hat{x})|x|}
$$


with $\alpha(\hat{x})(>0)$ independent of $p$, then for $x$ in $C$,

$$
|f(x)|<\operatorname{cst} \mathscr{P}(|x|) e^{-\alpha(\hat{x})|x|} .
$$

The next result applies in cases when $\tilde{f}$, rather than $f$, has support properties.

Theorem 3. If $\tilde{f}(p)=0$ in the sphere $S(P, r)$ of radius $r$ around a given point $P$ (i.e. in the region $|p-P|<r$ ), then $\forall \varepsilon>0, F$ satisfies at $P$ bounds of the form:

$$
|F(x, P ; \gamma)|<c_{\varepsilon}\left[(\gamma|x|)^{-v / 2} \mathscr{P}(|x|, \sqrt{\gamma|x|})\right] \exp \left\{-(1-\varepsilon) r^{2} \gamma|x|\right\}
$$

for all $\gamma>0$.

The following common corollary of Part (i) of Theorem 1 and of Theorem 3 will be directly useful in Sect. 3.

\section{Theorem 4.}

a) If $f=f^{\prime}+f^{\prime \prime}, f^{\prime}(x)=0$ in a cone $C$ and $\tilde{f}^{\prime \prime}(p)=0$ in $S(P, r), F$ satisfies at $P$ bounds of the form $(10)$ in each direction $\hat{x}$ of $C$ if $\gamma<d(\hat{x}) / 2 r$. More generally, the rate of fall-off in each direction of $C$ is at least equal (or arbitrarily close) to $\operatorname{Inf}\left[\frac{d(\hat{x})^{2}}{4 \gamma}, r^{2} \gamma\right]$, and in particular to $r d(\hat{x}) / 2$ if $\gamma=d(\hat{x}) / 2 r$.

b) Similarly, if $f=f_{1}^{\prime}+f_{1}^{\prime \prime}=f_{2}^{\prime}+f_{2}^{\prime \prime}=\cdots$ with $f_{i}^{\prime}(x)=0$ in a cone $C_{i}$ and $\widetilde{f}_{i}^{\prime \prime}(p)=0$ in $S\left(P, r_{i}\right), i=1,2, \ldots, F(x, P ; \gamma)$ decays exponentially in each direction $\hat{x}$ of $\bigcup_{i} C_{i}$. The rate of exponential fall-off is at least equal (or arbitrarily close) to the "enveloping function" of

$$
\operatorname{Sup}_{i}\left\{\operatorname{Inf}\left[\frac{d_{i}(\hat{x})^{2}}{4 \gamma}, r_{i}^{2} \gamma\right]\right\}
$$

Remarks.

1. Similar results apply if $f^{\prime}$ or $f_{i}^{\prime}$ does not vanish but decays exponentially in the directions of $C$ or $C_{i}$ as a consequence of part (ii) of Theorem 1.

2. Theorem 4 yields the following byproduct on the microsupport $\operatorname{ES}(\tilde{f})$ :

\section{Corollary 1}

$$
E S_{P}(\tilde{f}) \subset \text { complement of } \bigcup_{i} C_{i}
$$

3. The explicit result given in Theorem $4 \mathrm{~b}$ does imply somewhat improved results on rates of exponential fall-off. In particular, if $\gamma$ is very large, the result given in Theorem $4 \mathrm{~b}$ is

$$
\left[\operatorname{Sup}_{i} d_{i}(\hat{x})\right]^{2} / 4 \gamma,
$$

whereas one can establish a rate closer and closer (as $\gamma$ increases) to $d(\hat{x})^{2} / 4 \gamma$, where

6 The rate given below entails by itself a better rate of exponential fall-off (see Remark 3), called its "enveloping function" following a terminology proposed by analogy with "holomorphy envelopes" of analytic functions 

$d(\hat{x})$ is the distance to the complement of $\bigcup_{i} C_{i}$ and may be much larger than
$\operatorname{Sup}_{i} d_{i}(\hat{x})$.

4. It will be useful in Sect. 4 to consider a slightly different definition of the generalized Fourier transform (of any tempered distribution $\widetilde{f}$ ), namely

$$
F(\tau ; u, p, \gamma)=\int \tilde{f}\left(p^{\prime}\right) e^{-i \tau\left(p^{\prime} \cdot u\right)} e^{-\gamma \tau\left|p^{\prime}-p\right|^{2}} d p^{\prime},
$$

where $\tau$ is a scalar parameter that will tend to infinity. In view of the equality

$$
F(\tau ; u, p, \gamma)=F(\tau u, p ; \gamma /|u|)
$$

all previous results apply to $F(\tau ; \cdot)$ with $d(\hat{x})$ replaced by $d(u)$ and $|x|$ by $\tau$. More generally, rates $\alpha(u, p, \gamma)$ of exponential in $\tau$ of $F(\tau ; u, p, \gamma)$ satisfy the relation

$$
\alpha(u, p, \gamma)=\alpha(\hat{u}, p, \gamma /|u|)|u|
$$

which makes the link with rates of exponential in $|x|$ of $F(x, p ; \gamma)$.

\section{Macrocausality Properties of $\mathrm{N}$-Point Functions}

3.1 Preliminaries. For definiteness, we use the standard formulation of locality as a strict support property. In view of part (ii) of Theorem 1 of Sect. 2, results extend similarly to theories in which one assumes only exponential fall-off in space-like directions.

The connected chronological function $T\left(x_{1}, \ldots, x_{N}\right)$ (assumed for simplicity to be a well defined distribution even at coincident points) is the connected vacuum expectation value $\left\langle\Omega\left|\mathscr{T}\left(x_{1}, \ldots, x_{N}\right)\right| \Omega\right\rangle_{c}$ of the time-ordered product $\mathscr{T}$ of $N$ field operators $A\left(x_{1}\right), \ldots, A\left(x_{N}\right)$ (i.e. fields are ranged according to time components of $\left.x_{1}, \ldots, x_{N}\right)$. For any proper subset $I$ of indices among $1, \ldots, N$ ( $I$ non-empty and different from $(1, \ldots, N)), T_{I}\left(x_{1}, \ldots, x_{N}\right)$ is defined similarly with $\mathscr{T}\left(x_{1}, \ldots, x_{N}\right)$ replaced by $\mathscr{T}(x(I)) \mathscr{T}(x(J)), x(I)=\left\{x_{i}\right\}_{i \in I}, J=(1, \ldots, N) \backslash I$. Locality yields [5] the following "microcausal factorization" property ${ }^{7}$ of time-ordered products:

$$
\mathscr{T}\left(x_{1}, \ldots, x_{N}\right)=\mathscr{T}(x(I)) \mathscr{T}(x(J)) \quad \text { if } \quad x(I) \gtrsim x(J),
$$

where $x(I) \gtrsim x(J)$ means that $x(I)$ contains no point of $x(J)$ in its closed causal future: each $x_{j}, j \in J$ is space-like to, or is in the past cone of each $x_{i}, i \in I$. Equation (15) yields in turn

$$
\left(T-T_{I}\right)\left(x_{1}, \ldots, x_{N}\right)=0 \quad \text { if } \quad x(I) \gtrsim x(J) .
$$

This result is not by itself a causal property of $T$, since $T_{I}\left(x_{1}, \ldots, x_{N}\right) \neq T(x(J)) T(x(I))$, but will be directly useful, as in [5], when completed by the following support property [5] in energy-momentum space of $\widetilde{T}_{I}$, due to the spectral condition (and energy-momentum conservation)

$$
\begin{array}{rll}
\tilde{T}_{I}\left(p_{1}, \ldots, p_{N}\right)=0 & \text { if } & p_{1}+\cdots+p_{N} \neq 0 \\
\text { or } & \text { if } & p_{I} \notin V_{+}
\end{array}
$$

\footnotetext{
${ }^{7}$ A related property has also been proposed (see [14]) as a basic axiom under the name of microscopic causality
} 
where $p_{I}=\sum_{i \in I} p_{i}$. Moreover, in a theory with $\mu>0$ :

$$
\tilde{T}_{I}\left(p_{1}, \ldots, p_{N}\right)=0 \quad \text { if } \quad p_{I} \notin H_{+}(\mu) \cup V_{+}(2 \mu)
$$

and amputated functions

$$
\tilde{T}_{(I)}^{\mathrm{amp}}\left(p_{1}, \ldots, p_{N}\right)=\prod_{i=1}^{N}\left(p_{i}^{2}-\mu^{2}\right) \tilde{T}_{(I)}\left(p_{1}, \ldots, p_{N}\right)
$$

still satisfy (16-18) and moreover

$$
\tilde{T}_{I}^{\mathrm{amp}}\left(p_{1}, \ldots, p_{N}\right)=0 \quad \text { if } \quad p_{I} \notin V_{+}(2 \mu), \quad|I|=1 \quad \text { or } \quad N-1 .
$$

Macrocausality properties will be naturally expressed as exponential fall-off properties, as $\tau \rightarrow \infty$, of $\int T\left(x_{1}, \ldots, x_{N}\right) \prod_{i=1}^{N} \varphi_{i, \tau}\left(x_{i}\right) d x_{1} \cdots d x_{N}$ for suitable choices of the test functions $\varphi_{i, \tau}$. The simplest and best choice for present purposes is, up to normalization factors,

$$
\tilde{\varphi}_{i, \tau}\left(p_{i}^{\prime}\right)=e^{-\gamma \tau\left|p_{i}^{\prime}-p_{i}\right|^{2}} e^{i\left(p_{i}^{\prime} \cdot u_{i}\right) \tau}
$$

with given points $p_{i}, u_{i}$ and $\gamma>0$ : best results will be obtained for various values of $\gamma$ depending on the set of points $p_{i}, u_{i}$. In (21), $|p|^{2}=(p)_{0}^{2}+\vec{p}^{2}$. As $\tau \rightarrow \infty, \tilde{\varphi}_{i, \tau}$ is localized around $p_{i}$ up to exponential fall-off with a width of the order of $1 / \sqrt{\gamma \tau}$, whereas its Fourier transform, equal to

$$
\varphi_{i, \tau}\left(x_{i}\right)=\operatorname{cst}(\gamma \tau)^{-d / 2} e^{i p_{i} \cdot\left(x_{i}-\tau u_{i}\right)} e^{-\left|x_{i}-\tau u_{i}\right|^{2} /(4 \gamma \tau)},
$$

where $\left|x_{i}\right|^{2}=\left(x_{i}\right)_{0}^{2}+\vec{x}_{i}^{2}$, is localized around $x_{i}=\tau u_{i}$, up to exponential fall-off with a width of the order of $\sqrt{\gamma \tau}$, small compared to $\tau$, hence to the distances $\left|\tau u_{i}-\tau u_{j}\right|$ if $u_{i} \neq u_{j}$, at large $\tau$ and any given $\gamma$.

We note that the above integral of $T$ (or $T^{\mathrm{amp}}$ ) with the test functions $\varphi_{i, \tau}$ just mentioned is the generalized Fourier transform $F$ (or $F^{\mathrm{amp}}$ ) of $\tilde{T}$ (or $\tilde{T}^{\mathrm{amp}}$ ) as defined in Eq. (12) with $p=\left(p_{1}, \ldots, p_{N}\right), u=\left(u_{1}, \ldots, u_{N}\right)$ and $|p|^{2}=\sum_{i}\left|p_{i}\right|^{2}$. Other choices of the functions $\varphi_{i, \tau}$, of the form

$$
\tilde{\varphi}_{i, \tau}\left(p_{i}^{\prime}\right)=\chi_{i}\left(p_{i}^{\prime}\right) e^{-\gamma \tau\left|p_{i}^{\prime}-p_{i}\right|^{2}} e^{i\left(p_{i}^{\prime} \cdot u_{i}\right) \tau}
$$

with $\chi_{i}$ locally analytic around $p_{i}$ have their own interest and are also useful in the later analysis of $N$-particle collision amplitudes in Sect. 4. Exponential fall-off properties analogous to the above are then still satisfied up to some changes. The function $\varphi_{i, \tau}$ still decays exponentially like $\exp -\left|x_{i}-\tau u_{i}\right|^{2} /(4 \gamma \tau)$ in the region $\left|x_{i}-\tau u_{i}\right|<2 r_{i} \gamma \tau$, where $r_{i}$ is linked to the analyticity domain of $\chi_{i}$. Otherwise, a factor $\exp -r_{i}^{2} \gamma \tau$ is obtained. We state e.g. the following more precise result.

Lemma 1. If $\chi_{i}$ is a continuous function with compact support, locally analytic in the region $\left|p_{i}^{\prime}-p_{i}\right|<r_{i},\left|q_{i}^{\prime}\right|<r_{i}-\left|p_{i}^{\prime}-p_{i}\right|$ in complex space ( $p^{\prime}$ replaced by $\left.p^{\prime}+i q^{\prime}\right), \varphi_{i, \tau}$ satisfies bounds of the form

$$
\left|\varphi_{i, \tau}\left(\tau v_{i}\right)\right|<\operatorname{cst} \exp -\operatorname{Inf}\left[\frac{\left|v_{i}-u_{i}\right|^{2}}{4 \gamma}, r_{i}^{2} \gamma\right] \tau
$$


If $\chi$ is moreover $C^{\infty}$ (infinitely differentiable) everywhere, then, for any $\geqq 0$ integer $v$ and any $\gamma \geqq 0$,

$$
\left|\varphi_{i, \tau}\left(\tau v_{i}\right)\right|<\frac{c_{v}}{\left[1+\tau\left|u_{i}-v_{i}\right|\right]^{v}} \exp -\operatorname{Inf}\left[\frac{\left|v_{i}-u_{i}\right|^{2}}{4 \gamma}, r_{i}^{2} \gamma\right] \tau .
$$

Proof. Easy adaptation of results of [8].

Remark. The standard rapid fall-off of the Fourier transform of $\chi_{i}$, due to its $C^{\infty}$ character, is the particular case $\gamma=0$ of (25).

3.2 Main Results. For clarity, results of Theorem 5 (ii) are stated for test functions (21) which allow one to obtain the simplest and best results in this section. Results in other cases are indicated in Remark 4.

\section{Theorem 5.}

(i) Given test functions (21) or (23) (with $\chi_{i}$ locally analytic), $T\left(\left\{\varphi_{i, \tau}\right\}\right.$ ) decays exponentially, for any $\gamma>0$, in the $\tau \rightarrow \infty$ limit apart possibly from configurations $(u, p), u=\left(u_{1}, \ldots, u_{N}\right), p=\left(p_{1}, \ldots, p_{N}\right)$ of the set $\Sigma$ such that $\sum_{i=1}^{N} p_{i}=0$ and

(C1) Given any non empty set $I(\neq(1, \ldots, N))$ such that $u(I)$ contains no other point $u_{j}, j \notin I$, in its closed future, $p_{I}$ belongs to $V_{+}$, or in a theory with $\mu>0$ to $H_{+}(\mu) \cup V_{+}(2 \mu)$.

(ii) Given test functions of the form (21) and $(p, u) \notin \Sigma$, the rate of exponential fall-off in $\tau$ is at least equal (or arbitrarily close) to $\beta(u, p ; \gamma)$ for each $\gamma>0$, where $\beta$ is strictly positive and is determined as follows. For each $I(\neq(1, \ldots, N))$, let $d_{I}(u)$ be the distance of $u$ to the set of points $y$ such that $y(I)$ contains other points in its closed future, and let $r_{I}(p)$ denote the distance of $p$ to the set of points $p^{\prime}$ such that $\sum_{i=1}^{N} p_{i}^{\prime}=0$ and $p_{I}^{\prime} \in V_{+}$, or $p_{I}^{\prime} \in H_{+}(\mu) \cup V_{+}(2 \mu)$ in a theory with $\mu>0$. For each $\gamma$, let

$$
\beta_{I}(u, p ; \gamma)=\operatorname{Inf}\left[\frac{d_{I}(u)^{2}}{4 \gamma}, r_{I}^{2}(p) \gamma\right]
$$

Then:

$$
\beta(u, p ; \gamma)=\operatorname{Sup}_{I} \beta_{I}(u, p ; \gamma)
$$

If $\gamma$ is chosen equal to $d_{I}(u) /\left(2 r_{I}(p)\right)$ for some $I$ (such that $\left.d_{I}(u)>0, r_{I}(p)>0\right)$, then $\beta(u, p ; \gamma)$ is at least equal (or arbitrarily close) to $d_{I}(u) r_{I}(p) / 2$.

(iii) The same results apply to $T^{\mathrm{amp}}$, in a theory with mass $\mu>0$, with $H_{+}(\mu) \cup V_{+}(2 \mu)$ replaced moreover by $V_{+}(2 \mu)$ if $|I|=1$ or $N-1 ; \Sigma$ and $\beta$ are correspondingly replaced by $\Sigma^{\mathrm{amp}}$ and $\beta^{\mathrm{amp}}$.

Remarks.

1) Condition (C1) in (26) can be equivalently replaced, as easily seen, by $(\mathrm{C} 1)^{\prime}: p_{I} \in V_{-}$, or $p_{I} \in H_{-}(\mu) \cup V_{-}(2 \mu)$, if $u(I)$ has no other point in its closed causal past. (Here $V_{-}, H_{-}(\mu), V_{-}(2 \mu)$ are opposite to $V_{+}, H_{+}(\mu), V_{+}(2 \mu)$.) 
2) Results of (ii) imply moreover that the rate of exponential fall-off is at least equal (or arbitrarily close) to $\alpha(u, p ; \gamma)$, where $\alpha \geqq \beta$ is the "enveloping function" of $\beta$ (see Sect. 2).

3) Theorem 5 entails the following byproduct:

\section{Corollary 2.}

$$
E S\left(\tilde{T}^{(\mathrm{amp})}\right) \subset \Sigma^{(\mathrm{amp})} .
$$

4) In the case of test functions (23), rates of exponential fall-off are similar to above with a further restriction of the form $\beta_{I} \leqq r^{2} \gamma$, where $r$ is linked to the local analyticity domains of the functions $\chi_{i}$. Given a set $I$ (such that $r_{I}(p)>0, d_{I}(u)>0$ ), bounds on $\left|T\left(\left\{\varphi_{i, \tau}\right\}\right)\right|$ including both the exponential fall-off factor and a further rapid fall-off factor are obtained if the support of $\chi\left(p^{\prime}\right)=\prod \chi_{i}\left(p_{i}^{\prime}\right)$ lies in the region $\left|p^{\prime}-p\right|<r_{I}(p)$ (in which case the term $T_{I}\left(\left\{\varphi_{i, \tau}\right\}\right)$ vanishes).

Proof of Theorem 5 (outline). Theorem 1 (ii) is a particular case of Theorem $4 \mathrm{~b}$ of Sect. 2, obtained by writing $T^{(\mathrm{amp})}=\left(T-T_{I}\right)^{(\mathrm{amp})}+T_{I}^{(\mathrm{amp})}$ and using exponential fall-off properties due to Eqs. (16) and (17), (18), (20) respectively. In the case of test functions (23) (Remark 4), a similar argument allows one to treat the contribution $\left(T-T_{I}\right)^{(\mathbf{a m p})}\left(\left\{\varphi_{i, \tau}\right\}\right)$, using the convolution formula

$$
\left(T-T_{I}\right)^{(\mathrm{amp})}\left(\left\{\varphi_{i, \tau}\right\}\right)=\int\left(T-T_{I}\right)^{(\mathrm{amp})}\left(x^{\prime}\right) \prod_{i=1}^{N} \varphi_{i, \tau}\left(x_{i}^{\prime}\right) d x^{\prime},
$$

and Lemma 1 on $\varphi_{i, \tau}$. Theorem 5 (i) and Corollary 2 (Eq. (29)) are byproducts of previous results.

Physical Comments and Complements. The following results hold if $p$ is e.g. a physical point of a given process in a massive theory $\left(\Sigma p_{i}=0, p_{i}^{2}=\mu^{2}, i=1, \ldots, N\right.$, $\left(p_{i}\right)_{0}<0$ if $i$ is initial, $\left(p_{i}\right)_{0}>0$ if $i$ is final). Conditions (C2) (C3) are essentially contained in [5] (in a less explicit form).

(i) Let $(p, u)$ belong to the "causal" set $\Sigma$. Then

(C2) The set $u\left(I_{f}\right)$ of final points is contained in the closed causal future of the set $u\left(I_{\text {in }}\right)$ of initial points (and $u\left(I_{\text {in }}\right)$ is contained in the closed past of $u\left(I_{f}\right)$ ).

$(\mathrm{C} 2)^{\prime}$ Any subset $u\left(I_{\text {in }}^{\prime}\right)$ of initial points $\left(I_{\text {in }}^{\prime} \subset I_{\text {in }}\right)$ must contain final points in its closed causal future (and any subset of final points must contain initial points in its closed past).

Condition $(\mathrm{C} 2)^{\prime}$ is e.g. needed so that the total energy-momentum $p_{I}$, where $I$ is the union of $I_{\text {in }}^{\prime}$ and of all other (initial or final) indices $j$ such that $x_{j}$ belongs to the closed future of $u\left(I_{\text {in }}^{\prime}\right)$, will belong to $V_{+}$. The result is in agreement with the idea (see Sect. 1) that energy-momentum can propagate only in future cones (in an asymptotic sense): the energy-momentum outgoing at final points in the future of $u\left(I_{\text {in }}^{\prime}\right)$ will compensate at least the energy-momentum incoming at points of $u\left(I_{\text {in }}^{\prime}\right)$. The more detailed condition $p_{I} \in H_{+}(\mu) \cup V_{+}(2 \mu)$ in $(\mathrm{C} 1)$, in a theory with $\mu>0$, indicates that, for connected functions, there cannot be strict compensation unless $I_{\text {in }}^{\prime}=I_{\text {in }}$ (in which case $I=(1, \ldots, N)$ and $p_{I}=0$ ): the energy-momentum outgoing at final points in the future of $u\left(I_{\text {in }}^{\prime}\right)$ must also compensate part of the energy-momentum incoming at other initial points which do not belong to $u\left(I_{\text {in }}^{\prime}\right)$ but lie in the past of these final points. 
(ii) Let $(p, u)$ belong to $\Sigma^{\text {amp }}$. Then $(\mathrm{C} 2)(\mathrm{C} 2)^{\prime}$ are satisfied and moreover:

(C3) "Extremal" initial or final points cannot be isolated: at least two or more such points lie at the same position in space-time. (An initial point $u_{i}$ is "extremal" if there is no other initial point in its closed past unless both lie at the same position. Similar definition for final points with closed future replacing closed past.)

Physical interpretation, e.g. for initial points: at least two initial particles must first interact if there are to be interactions.

Proof of (C3). Assume a final point $u_{f}$ is extremal. If there is no initial point in its closed future, let $I$ be reduced to the single index $f$. Since there is no point in the closed future of $u_{f}=u(I), p_{I}=p_{f}$ should belong to $V_{+}(2 \mu)$, whereas $p_{f} \in H_{+}(\mu)$. If there are initial points in the closed future of $u_{f}$ (which must lie, as a matter of fact, at the same position as $u_{f}$ in view of (C2)), let $I$ be the set composed of $f$ and of corresponding initial indices. Then $p_{I}$ is the sum of $p_{f} \in H_{+}(\mu)$ and of other energy-momenta in $H_{-}(\mu)$, so that $p_{I}$ cannot belong to $H_{+}(\mu) \bigcup V_{+}(2 \mu)$. Q.E.D.

We now complete Theorem 5 with the following more refined result on $T$ in a theory with $\mu>0$. For simplicity, we do not try here to specify rates of exponential fall-off and thus restrict ourselves in Theorem 6 (ii) to a statement on the (microlocal, analytic) essential support of $\widetilde{T}$, which ensures exponential fall-off of $T\left(\left\{\varphi_{i, \tau}\right\}\right)$, for test functions (21) or (23) and any $\gamma>0$, for points $u$ outside $\Sigma^{\prime}(p)$. Part (i) of Theorem 6, reobtained below, is well known by other methods in field theory.

Theorem 6. Given any physical point $P$,

(i)

$$
\tilde{T}\left(p_{1}, \ldots, p_{N}\right)=\prod_{i=1}^{N}\left[p_{i}^{2}-\mu^{2}+i \varepsilon\right]^{-1} \tilde{T}^{\mathrm{amp}}\left(p_{1}, \ldots, p_{N}\right)
$$

in the neighborhood of $P$.

(ii) $E S_{P}(\tilde{T}) \subset \Sigma^{\prime}(P)=\left\{x=\left(x_{1}, \ldots, x_{N}\right)\right.$; there exist $y=\left(y_{1}, \ldots, y_{N}\right)$ and scalars $\lambda_{1}, \ldots, \lambda_{N}$, $\lambda_{i} \geqq 0$, such that $x_{i}-y_{i}=\lambda_{i} P_{i}, \forall i$, and $\left.(y, P) \in \Sigma^{\mathrm{amp}}\right\}$.

The various results on possible "causal" configurations with respect to $T^{\mathrm{amp}}$ and $T\left((p, u) \in \Sigma\right.$ amp or $\left.(p, u) \in \Sigma^{\prime}\right)$ are illustrated in Fig. 1.

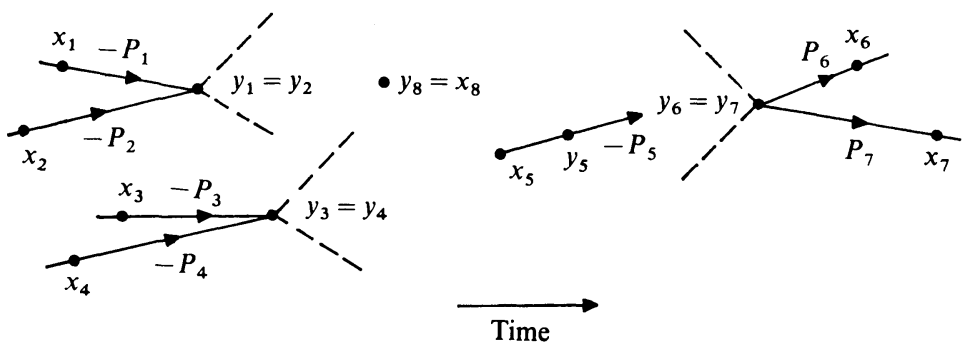

Fig. 1. Possible causal configurations $\left(x_{1}, \ldots, x_{8}\right)$ and $\left(y_{1}, \ldots, y_{8}\right)$ relative to non-amputated and amputated functions at a physical point $\left(P_{1}, \ldots, P_{8}\right), 1, \ldots, 5$ initial, 6, 7, 8 final (and $\left.P_{5}+P_{6}+P_{7} \in H_{+}(\mu) \cup V_{+}(2 \mu)\right)$ 
Proof of Theorem 6 (outline). At e.g. a physical point $P$, one checks easily that

$$
\begin{aligned}
& E S_{P}\left(\sum_{i=1}^{N}\left(p_{i}^{2}-\mu^{2}+i \varepsilon\right)^{-1}\right) \subset\{x=\left(x_{1}, \ldots, x_{N}\right), x_{i}=\lambda_{i} P_{i}, \\
&\left.\lambda_{i}<0 \text { if } i \text { is initial, } \lambda_{i}>0 \text { if } i \text { is final }\right\} .
\end{aligned}
$$

Points in the set defined in the right-hand side of (33) cannot be opposite to points in $E S_{P}\left(\widetilde{T}^{\mathrm{amp}}\right.$ ) (in view e.g. of condition $(\mathrm{C} 2)$ ) so that standard "non- $u=0$ " results of [9] can be directly applied to define the product in the right-hand side of (31) and to show that its essential support is contained in the set (32). It remains to show the equality (31). In view of the definition of $\tilde{T}^{\mathrm{amp}}$, the product of $\prod_{i=1}^{N}\left(p_{i}^{2}-\mu^{2}\right)$ with the difference of the two sides of (31) vanishes, so that this difference is at most a sum of products involving mass-shell $\delta$-functions. Such terms cannot be present because their essential support would be inconsistent with those of each term of the difference (and hence of the difference itself).

3.3 Space-Time Cluster Property. The purpose of this sub-section is to establish (part (iii) of Theorem 7) the exponential fall-off, in a theory with $\mu>0$, of $T\left(x_{1}, \ldots, x_{N}\right)$ itself, or more precisely of any regularized function $T_{\varphi}=T * \varphi$ obtained by convolution with a $C^{\infty}$ function $\varphi$ with (small) compact support around the origin, for large space-like separation of subgroups of points. For simplicity, we consider two groups $x(I), x(J), J=(1, \ldots, N) \backslash I . F$ denotes below the generalized Fourier transform of $\widetilde{T}$ in the sense of Eq. (1).

\section{Theorem 7.}

(i) Whatever $p=\left(p_{1}, \ldots, p_{N}\right)$ is, $x=\left(x_{1}, \ldots, x_{N}\right)$ is non-causal at $p((x, p) \notin \Sigma)$ if $x(I) \sim x(J)$, i.e. $x(I)$ and $x(J)$ space-like $\left(x_{i}-x_{j}\right.$ space-like, $\left.\forall i \in I, j \in J\right)$.

(ii) Given a configuration $\hat{x}(|\hat{x}|=1)$ such that $\hat{x}(I) \sim \hat{x}(J)$, the rate of exponential fall-off in the direction of $\hat{x}$ is at least equal (or arbitrarily close) to $c \mu d(\hat{x}), \forall p$, if $\gamma=d(\hat{x}) /(4 c \mu)$ where

$$
d(\hat{x})=\operatorname{Min}\left(d_{I}(\hat{x}), d_{J}(\hat{x})\right)>0,
$$

and $c$ is a fixed $>0$ constant (independent of $p$ and $\hat{x}$ ) such that

$$
\operatorname{Sup}\left(r_{I}(p), r_{J}(p)\right) \geqq 2 c \mu, \quad \forall p .
$$

(iii) $T_{\varphi}\left(x_{1}, \ldots, x_{N}\right)$ decays exponentially as $|x| \rightarrow \infty$ with a rate of fall-off at least equal (or arbitrarily close) to $\operatorname{c\mu d}(\hat{x})$.

Proof.

(i) Both $x(I)$ and $x(J)$ have no other point in their closed future, but at least $p_{I}$ or $p_{J}\left(\right.$ depending on $p$ ) does not belong to $H_{+}(\mu) \cup V_{+}(2 \mu)$ if $\Sigma p_{k}\left(=p_{I}+p_{J}\right)=0$.

(ii) From results of Sect. 3.2. The existence of $c>0$ is easily checked. On the other hand, both $d_{I}(\hat{x})$ and $d_{J}(\hat{x})$, hence $d(\hat{x})$, are $>0$ if $\hat{x}(I) \sim \tilde{x}(J)$.

(iii) In a non-rigorous way, one may write (for any given $\gamma>0$ )

$$
T\left(x_{1}, \ldots, x_{N}\right)=(\gamma|x|)^{d / 2} \int d p_{1} \cdots d p_{N} F(x, p ; \gamma)
$$

as checked formally by interchange of orders of integrations. The exponential 
fall-off of $T$ thus arises from the minimal uniform fall-off, as $p$ varies, provided by part (ii) (with suitable $\gamma$ ). To make this proof rigorous, it is sufficient to check that Theorem 2 of Sect. 2 can be applied to $T_{\varphi}=\left(T-T_{I}\right)_{\varphi}+\left(T_{I}\right)_{\varphi}=$ $\left(T-T_{J}\right)_{\varphi}+\left(T_{J}\right)_{\varphi}$, i.e. to obtain bounds including a uniform exponential fall-off factor in $|x|$ and a further factor with sufficient decrease as $|p| \rightarrow \infty$. To that purpose, $\left(T-T_{I}\right)_{\varphi}$ and $\left(T-T_{J}\right)_{\varphi}$ are treated by part (i)' of Theorem 1 of Sect. 2. Remaining terms are easily treated (for e.g. $|x|>1$ ) by noting that $r_{I}(p)$ and $r_{J}(p)$ tend to infinity (at least linearly) as $|p| \rightarrow \infty$ in respective sectors in $p$-space.

\section{Macrocausality Properties of $\boldsymbol{N}$-Particle Collision Amplitudes}

4.1 General Method and Preliminary Results. In view of the standard link [3], recalled in Sect. 1.1, between $\widetilde{T}^{\mathrm{amp}}$ and the connected $S$ matrix, connected collision amplitudes between initial and final wave functions $\hat{\varphi}_{i, \tau}$ that are mass-shell restrictions of the functions (21) or (23) can be written

$$
S^{c}\left(\left\{\hat{\varphi}_{i, \tau}\right\}\right)=\int \tilde{T}^{\mathrm{amp}}\left(p^{\prime}\right) \delta_{M}\left(p^{\prime}\right) \chi\left(p^{\prime}\right) e^{-i\left(p^{\prime} \cdot u\right) \tau} e^{-\gamma \tau\left|p^{\prime}-p\right| 2} d p^{\prime},
$$

where $p \cdot u=\sum \varepsilon_{i} p_{i} \cdot u_{i}\left(\varepsilon_{i}=-1\right.$ if $i$ is initial, $\varepsilon_{i}=+1$ if $i$ is final), $\chi(p)=\prod_{i} \chi_{i}\left(p_{i}^{\prime}\right)$,

$$
\delta_{M}\left(p^{\prime}\right)=\prod_{i=1}^{N} \delta\left(p_{i}^{\prime 2}-\mu^{2}\right) \theta\left(\varepsilon_{i}\left(p_{i}^{\prime}\right)_{0}\right)
$$

and where the product $\tilde{T}^{\mathrm{amp}} \delta_{M}$ is a well defined distribution, as established so far away from $M_{0}$ points such that two initial or two final energy-momenta are colinear (i.e. equal in a theory with only one mass $\mu$ ). We below either consider test functions $\chi_{i}$ with supports that exclude $M_{0}$ points or make a regularity assumption to cover these points.

We first present a semi-heuristic analysis of the way exponential fall-off properties of $S^{c}\left(\left\{\hat{\varphi}_{i, \tau}\right\}\right)$ can be established from those of $N$-point functions. Given any $\eta$ such that $0<\eta<1$, the integral (37) can be considered as the Fourier transform (at $x=\tau u$ ) of the product

$$
\left[\tilde{T}^{\mathrm{amp}}\left(p^{\prime}\right) e^{-\eta \gamma \tau\left|p^{\prime}-p\right|^{2}}\right] \times\left[\chi\left(p^{\prime}\right) \delta_{M}\left(p^{\prime}\right) e^{-(1-\eta) \gamma \tau\left|p^{\prime}-p\right|^{2}}\right]
$$

so that $S^{c}\left(\left\{\hat{\varphi}_{i, \tau}\right\}\right)$ can be expressed as the convolution integral

$$
S^{c}\left(\left\{\hat{\varphi}_{i, \tau}\right\}\right)=\int F^{\mathrm{amp}}(\tau ; v, p, \eta \gamma) \prod_{i=1}^{N} f_{i, \tau}\left(u_{i}-v_{i}, p_{i},(1-\eta) \gamma\right) d(\tau v),
$$

where $F^{\mathrm{amp}}$ is the generalized Fourier transform of $\tilde{T}^{\mathrm{amp}}$ in the sense of Eq. (12), and is thus the action of $T^{\mathrm{amp}}$ on test functions of the form (21) (see Sect. 3), and

$$
f_{i, \tau}\left(u_{i}, p_{i} ; \gamma\right)=\int \chi_{i}\left(p_{i}^{\prime}\right) e^{-\gamma \tau\left|p_{\imath}^{\prime}-p_{\imath}\right|^{2}} e^{-i p_{i}^{\prime} \cdot u_{i} \tau} \delta_{+}\left(p_{i}^{\prime 2}-\mu^{2}\right) d p_{i}^{\prime}
$$

If, given $(p, u)$, there is $a>0$ uniform lower bound on the rate of exponential fall-off in $\tau$ obtained, as $v=\left(v_{1}, \ldots, v_{N}\right)$ varies, from the various factors $F^{\text {amp }}$ and $f_{i, \tau}$ in the integrand of (39), $S^{c}\left(\left\{\hat{\varphi}_{i, \tau}\right\}\right)$ can then be expected, modulo convergence problems in $v$, to decay exponentially in $\tau$ with at least the uniform rate thus determined. We give in Sect. 4.2 a slightly weaker result but first give the following preliminary 
result on exponential fall-off properties of the functions $f_{i, \tau}$, which generalizes that given in [4] in a particularly simple situation and is a more precise and complete version of that given in [13]. We consider for simplicity a fixed point $p_{i}$ and leave the index $i$, as also the dependence of results on $p_{i}$, implicit.

Theorem 8. (i) $\left|f_{\tau}(u ; \gamma)\right|$ decays exponentially as $\tau \rightarrow \infty$ if $u$ does not lie on the trajectory $(0, p)$ passing through the origin and parallel to $p$.

(ii) The rate of exponential fall-off in $\tau$ satisfies the properties indicated in Theorem 10 in Appendix 1 . If $\chi \equiv 1$, it is moreover at least equal to cst $\alpha(u ; \gamma)$, with a constant independent of $u, \gamma$, and

$$
\begin{array}{ll}
\alpha(u ; \gamma)=d(u ;(0, p))^{2} /(4 \gamma) & \text { if u is space - like, } \\
\alpha(u ; \gamma)=\operatorname{Min}\left[d(u ;(0 ; p))^{2} / 4 \gamma, \rho(p, \hat{u})^{2} \gamma\right] & \text { if } u \text { is time - like, }
\end{array}
$$

where $d(u ;(0, p))$ is the (euclidean) distance of $u$ to the trajectory $(0, p), \hat{u}$ is the unit vector in the direction of $u$ and $\rho(p, \hat{u})$ is the (euclidean) distance of $p$ to the point $p^{\prime}$ of $H_{+}(\mu)$ in the direction of $\hat{u}$.

(iii) If $\chi$ is not the function $1, \alpha_{\chi}(u, p ; \gamma)$ is moreover less than $r(\chi)^{2} \gamma$, where $r(\chi)$ is linked to the analyticity domain of $\chi$ around $p$. If $\chi$ is $C^{\infty}$ with compact support and if $u$ lies outside the velocity cone $V(\chi)$ (set of trajectories $\left(0, p^{\prime}\right)$ for all point $p^{\prime}$ of $H_{+}(\mu)$ in the support of $\left.\chi\right)$, bounds on $\left|f_{\tau}(u ; \gamma)\right|$ include also a further rapid fall-off factor $c_{v}(\hat{u}) /(1+\tau|u|)^{v}, \forall$ integer $v \geqq 0$, where $c_{v}$ may tend to zero as $\hat{u}$ approaches $V(\chi)$.

Remark. The standard rapid fall-off outside $V(\chi)$ of the space-time wave function associated to $\chi$ is the particular case $\gamma=0$ of the last part of Theorem 8 .

Proof of Theorem 8 (outline). Theorem 8 is a consequence of the assumed local analyticity of $\chi$. The latter allows suitable local distortions of the integration domain in (40) (i.e. the real mass-shell $H_{+}(\mu)$ ) in the complex mass-shell, in the line of the ideas and methods developed in [8] in a simpler situation. The local distortion is restricted both by the analyticity domain of $\chi_{i}$ around $p_{i}$ and by the geometry of the mass-shell. The result is in agreement with general results on rates of exponential fall-off of generalized Fourier transforms (see App. 1.1) and gives more precise information in the case under consideration.

4.2 Main Result and Complements. The result stated in Theorem 9 below allows one to derive exponential fall-off properties of connected amplitudes $S^{c}\left(\left\{\hat{\varphi}_{i, \tau}\right\}\right)$, for functions $\hat{\varphi}_{i, \tau}$ that are mass-shell restrictions of functions (21) or (23), from preliminary information relative to $T^{\mathrm{amp}}$ for functions of the form (21). It applies in particular if one starts from results on $T^{\mathrm{amp}}$ of Sect. 3 but may also apply more generally if one starts from improved information relative to $T^{\text {amp }}$ (obtained e.g. from assumptions going beyond locality and spectrum).

The proof of part (i) relies on the condition that $E S_{p}\left(\tilde{T}^{\text {amp }}\right)$ contains no (non-zero) point of $-E S_{p}\left(\delta_{M}\right)$, i.e. of the form $\left\{\lambda_{i} p_{i}\right\}$, where $\lambda_{i}$ is an arbitrary real scalar. This condition is satisfied in Sect. 3 if $p$ is not a $M_{0}$ point in view e.g. of Condition (C3). (The situation at $M_{0}$ points will be discussed later.) On the other hand, the proof of part (ii), makes use of results relative to $T^{\text {amp }}$ not only for functions of the form (21) but also of the form (23): needed results for functions (23), including rapid fall-off factors, are either known (they are directly established 
from locality and spectrum in Sect. 3: see Remark 4 in Sect. 3.2) or can be derived from those applying to functions (21) through results of [9], as in App. 1.2.

\section{Theorem 9.}

(i) Given test functions (21) or (23), $S^{c}\left(\left\{\hat{\varphi}_{i, \tau}\right\}\right)$ decays exponentially in the $\tau \rightarrow \infty$ limit for any $\gamma>0$, apart possibly from configurations $(p, u)$ such that either $p \in M_{0}$ or $u$ belongs to the set

$$
\begin{aligned}
\hat{\Sigma}(p)=\{u ; \exists y= & \left(y_{1}, \ldots, y_{N}\right) \in E S_{p}\left(\tilde{T}^{\text {amp }}\right) \text { and } \\
& \text { real scalars } \left.\lambda_{1}, \ldots, \lambda_{N} \text { such that } u_{i}-y_{i}=\lambda_{i} p_{i}, i=1, \ldots, N\right\} .
\end{aligned}
$$

(ii) The rate of exponential fall-off in $\tau$, for any $\gamma>0$, if $p \notin M_{0}$ and $u \notin \hat{\Sigma}(p)$, is at least equal, up to a fixed (multiplicative) constant, to the $(>0)$ minimal rate obtained as $v$ varies, from the various factors $F^{\mathrm{amp}}$ and $f_{i, \tau}$ in the integrand of (39).

\section{Remarks.}

1. The fixed $(<1)$ multiplicative constant that occurs in (ii) is due to technical problems in the proof.

2. $\hat{\Sigma}(p)$ is defined in the same way as $\Sigma^{\prime}(p)$ in (32) except that the constraints on the signs of $\lambda_{i}$ are removed. If one starts from locality and the spectral condition (with mass $\mu>0$ ), possible causal configurations $u$ can be illustrated as in Fig. 1, except that each $u_{i}$ may lie on the full trajectory $\left(y_{i}, p_{i}\right)$ parallel to $p_{i}$ and passing through $y_{i}$.

3. The fact that the rate obtained in (ii) is $>0$ can be seen as follows. If $v$ is causal with respect to $T^{\mathrm{amp}}$, the term $F^{\mathrm{amp}}(\tau ; v, p, \eta \gamma)$ provides no exponential fall-off factor in $\tau$, but at least one of the terms $f_{i, \tau}\left(u_{i}-v_{i} ;(1-\eta) \gamma\right)$ produces such a factor: one or more $v_{i}$ does not belong to the trajectory $\left(p_{i}, u_{i}\right)$. On the other hand, if each $v_{i}$ belongs to the trajectory $\left(p_{i}, u_{i}\right), v$ is non-causal with respect to $T^{\text {amp }}$, so that the term $F^{\mathrm{amp}}$ produces an exponential fall-off factor. If $p$ is not a $M_{0}$ point, there is $a>0$ minimal rate as $v$ tends to infinity in any direction. In fact, if some $v_{i}$ tend to infinity in the direction of the corresponding $p_{i}$ (or in directions close to it), $v$ is non-causal with respect to $T^{\mathrm{amp}}$ and a minimal $>0$ rate of exponential fall-off is obtained for $F^{\mathrm{amp}}$. If some $v_{i}$ tend to infinity in other directions (not close to the direction of $p_{i}$ ), a minimal $>0$ rate is obtained for $f_{i, \tau}$.

Proof of Theorem 9 (outline). Theorem 9 is a particular case of Theorem 11 in Appendix 1.2. We briefly give below an idea of the proof in the physical situation (with some slight specific changes). Suitable partitions of unity, of the form $1=\psi\left(p^{\prime}\right)+(1-\psi)\left(p^{\prime}\right), 1=\psi_{i}\left(p_{i}^{\prime}\right)+\left(1-\psi_{i}\right)\left(p_{i}^{\prime}\right)$ are introduced, with $C^{\infty}$ functions $\psi, \psi_{i}$ that have sufficiently small supports around $p=\left(p_{1}, \ldots, p_{N}\right)$ or $p_{i}$ respectively, and are equal to one locally. $S^{c}\left(\left\{\hat{\varphi}_{i, \tau}\right\}\right)$ is then written as the sum of a contribution analogous to (39), except that $\widetilde{T}^{\text {amp }}$ and $\chi_{i}$ in the definitions of $F^{\text {amp }}$ and $f_{i, \tau}$ are replaced by $\widetilde{T}^{\mathrm{amp}}\left(p^{\prime}\right) \psi\left(p^{\prime}\right)$ and $\chi_{i}\left(p_{i}^{\prime}\right) \psi_{i}\left(p_{i}^{\prime}\right)$ respectively, and remaining terms. Rapid fall-off factors in the analysis of the first contribution (see comments preceding the statement of Theorem 9) will ensure convergence in $v$. The exponential decay of remaining contributions follows from the vanishing of $1-\psi$, or $1-\psi_{i}$, is the neighborhood of $p$, or $p_{i}$.

Situation at $M_{0}$ Points. If $p$ is a $M_{0}$ point, the proof outlined above does not 


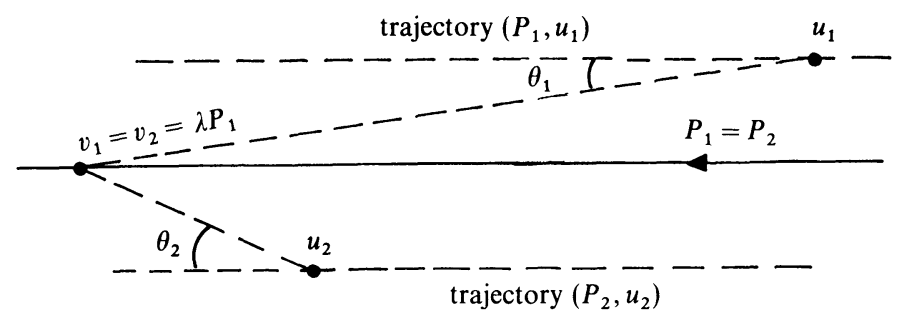

Fig. 2. $\left(P_{1}, P_{2}\right.$ initial, $\left.P_{1}=P_{2} ; \lambda>0\right)$

apply. However, a minimal $>0$ rate of exponential fall-off in $\tau$, as $v$ varies, can still be extracted as above from the integrand of (39) apart from configurations $u$ of a set $\hat{\Sigma}^{\prime}(p)$ analogous to $\hat{\Sigma}(p)$ except that there is no constraint on $u_{i}$ if there is one or more $p_{j}$ equal to $p_{i}(j \neq i)$. If $u \in \hat{\Sigma}^{\prime}$, the existence of a minimal $>0$ rate is not guaranteed in view of possible sequences of points $v$ such that $v_{i}$ tends to infinity in the direction of $p_{i}$ whenever $p_{i}$ is equal to some other $p_{j}$, and such that the rate of exponential fall-off in $\tau$ of $F^{\text {amp }}$ vanishes, at least in the limit; rates of fall-off in $\tau$ of the functions $f_{i, \tau}$ are in this case either zero or tend to zero in the limit because the factor $\rho\left(p_{i}, \hat{v}_{i}-u_{i}\right)$ in (42) tends to zero: see the example of Fig. 2 in which the rates of exponential fall-off of $f_{1, \tau}\left(v_{1}-u_{1}\right)$ and $f_{2, \tau}\left(v_{2}-u_{2}\right)$ are non-zero but become arbitrarily small as $\lambda \rightarrow \infty\left(\rho \rightarrow 0\right.$ as $\theta_{1}$, or $\left.\theta_{2}, \rightarrow 0\right)$.

The following property can be conjectured and might be established modulo some regularity assumptions.

Conjecture. If $u \notin \hat{\Sigma}^{\prime}(p), S^{c}\left(\left\{\hat{\varphi}_{i, \tau}\right\}\right)$ decays exponentially in $\tau$ with a $(>0)$ rate of fall-off determined as in part (ii) of Theorem 9.

Lower bounds of physical interest on rates of exponential fall-off provided by Theorem 9 , or by the conjecture just mentioned at $M_{0}$ points, can be established in various situations. A simple situation, analogous to that considered in [4] and to one of those considered in [7], is treated in Appendix 3.

\section{Appendix 1: Complementary Mathematical Results}

\subsection{Exponential Fall-Off Properties of Generalized Fourier Transforms: General} Results. Proofs of results below, which complement those of $[8,9]$, are omitted for conciseness and will be given elsewhere.

Let $\tilde{f}$ be any tempered distribution in $\mathbb{R}^{n}$ with microsupport $\operatorname{ES}(\tilde{f})$ : given $p, u$, with e.g. $|u|=1$, such that $u \notin E S_{p}(\widetilde{f})$, it is known by definition of $E S_{p}(\tilde{f})$ (see [9]) that $F(\tau u, p ; \gamma)$, as defined in Eq. (1), decays exponentially with $\tau$ for all sufficiently small values of $\gamma>0$ with a rate at least proportional to $\gamma$ at small $\gamma$. We define $d(p, u)=\left(\right.$ euclidean) distance of $u$ to $E S_{p}(\tilde{f}) ; \rho(p, u)=\operatorname{Sup}_{\rho^{\prime} \geqq 0}\left\{\rho^{\prime} ; u \notin E S_{p^{\prime}}(\tilde{f}), \forall p^{\prime}\right.$ such that $\left.\left|p^{\prime}-p\right|<\rho^{\prime}\right\}$, with $\rho(p, u)>0$ since $u \notin E S_{p}(\tilde{f})$ (see [9]). We then state

Theorem 10.

(i) $F$ decays exponentially as $\tau \rightarrow \infty$ for any $\gamma>0$. 
(ii) $\forall \eta>0,0<\eta<1$, the rate of exponential fall-off of $F(\tau u, p ; \gamma)$ in $\tau$ is at least equal to

$$
[d(p, u)(1-\eta)]^{2} / 4 \gamma
$$

for all sufficiently large values of $\gamma\left(\gamma>\gamma_{0}^{\prime}(p, u, \eta)\right.$, where $\gamma_{0}^{\prime}$ may tend to infinity as $\eta \rightarrow 0)$.

(iii) $\forall \rho^{\prime}<\rho(p, u)$, the rate of exponential fall-off of $F$ in $\tau$ is at least equal to $\rho^{\prime 2} \gamma$ for all sufficiently small $\gamma\left(\gamma<\gamma_{0}^{\prime \prime}\left(p, u, \rho^{\prime}\right)\right)$.

\section{More generally}

(iv) $\forall \eta, 0<\eta<1$, there exists $\alpha(p, u, \eta)>0$ such that, given any $\alpha^{\prime}<\alpha(p, u, \eta)$, the rate of exponential fall-off of $F$ in $\tau$ is at least equal to $\alpha^{\prime} \gamma$ for $\gamma \leqq \frac{1}{2 \sqrt{\alpha^{\prime}}} d(p, u)(1-\eta)$. It is at least equal, for any $\gamma>0$, to

$$
\operatorname{Sup}_{\eta ; 0<\eta<1}\left\{\operatorname{Inf}\left[\frac{(d(p, u)(1-\eta))^{2}}{4 \gamma}, \alpha(p, u, \eta) \gamma\right]\right\} .
$$

Remark. In general, $\alpha(p, u, \eta)<\rho(p, u)^{2}, \alpha(p, u, \eta) \rightarrow \rho(p, u)^{2}$ as $\eta \rightarrow 1$ and $\alpha(p, u, \eta) \rightarrow 0$ as $\eta \rightarrow 0$.

Sections 2 and 3 present particular cases of interest in which more precise results, consistent with those of Theorem 10 , are obtained.

\subsection{A Convolution Theorem. Let $\tilde{f}_{1}, \tilde{f}_{2}$ be tempered distributions on $\mathbb{R}^{n}$ such that}

$$
E S_{p}\left(\tilde{f}_{1}\right) \cap E S_{p}\left(\tilde{f}_{2}\right)=\phi
$$

(apart from the origin) at a given point $p$. The product $\tilde{f}_{1} \tilde{f}_{2}$ is then well defined locally and $E S_{p}\left(\tilde{f}_{1} \widetilde{f}_{2}\right)$ satisfies the relation (see e.g. [9])

$$
E S_{p}\left(\tilde{f}_{1} \tilde{f}_{2}\right) \subset\left\{u ; u=u_{1}+u_{2} ; u_{1} \in E S_{p}\left(\tilde{f}_{1}\right), u_{2} \in E S_{p}\left(\tilde{f}_{2}\right)\right\},
$$

where $E S_{p}$ is understood as a closed cone with apex at the origin.

We assume below that the product $\tilde{f}_{1} \widetilde{f}_{2}$ is also well defined everywhere. Given $p$, left implicit below, let $\alpha_{1}(u, \gamma), \alpha_{2}(u, \gamma)$ be rates of exponential fall-off in $\tau$ of $F_{1}(\tau ; u, p, \gamma)$ and $F_{2}(\tau ; u, p, \gamma)$ respectively, or possibly lower (continuous) bounds on the latter ${ }^{8}$. Actual rates are known to satisfy in particular the following properties: $\alpha_{\sigma}>0$ if $u \notin E S_{p}\left(\tilde{f}_{\sigma}\right), \sigma=1,2$ and, on the other hand (see Appendix 1.1 and (14)), $\alpha_{\sigma}(u, \gamma)$ is close to $\rho_{\sigma}(p, \hat{u})^{2} \gamma$ when $\gamma /|u|$ is small. (In contrast to Appendix 1.1, $|u|$ may be different from 1.) These properties will be assumed also on possible lower bounds. We then state

Theorem 11. Let $u$ be any given point outside the right-hand side of (45). Given $u$ and $\gamma>0$, the rate of exponential fall-off in $\tau$ of $F(\tau ; u, p, \gamma)$ is at least equal to $c$ $\alpha(u, \gamma)$, where $c$ is a fixed constant $(\leqq 1)$ independent of $u, p, \gamma$ and $\alpha(u, \gamma)>0$, where

$$
\alpha(u, \gamma)=\operatorname{Min}_{v}\left[\alpha_{1}\left(v, \frac{\gamma}{2}\right)+\alpha_{2}\left(u-v, \frac{\gamma}{2}\right)\right]
$$

\footnotetext{
${ }^{8}$ Namely, e.g. $F_{1}$ is assumed to satisfy bounds including the exponential fall-off factor $\exp -\alpha_{1}(u, \gamma) \tau$ and further factors such as those given in (2), (10)
} 
Remark. The constant $c$ can probably be chosen equal to one. The proof given below introduces some technical losses and gives $c<1$. A slightly improved version of Theorem 11 can be obtained on the other hand with $\frac{\gamma}{2}$ replaced by $\eta \gamma$ and $(1-\eta) \gamma$ in $\alpha_{1}$ and $\alpha_{2}$ respectively.

Proof (Outline). By writing $\tilde{f}_{1} \tilde{f}_{2}\left(p^{\prime}\right) e^{-\gamma \tau\left|p^{\prime}-p\right|^{2}}$ in the form $\tilde{f}_{1}\left(p^{\prime}\right) e^{-(\gamma / 2) \tau\left|p^{\prime}-p\right|^{2}} \times$ $\tilde{f}_{2}\left(p^{\prime}\right) e^{-(\gamma / 2) \tau\left|p^{\prime}-p\right|^{2}}, F$ can be written at least formally as the convolution product

$$
F(\tau ; u, \gamma)=\int F_{1}\left(\tau ; v, \frac{1}{2} \gamma\right) F_{2}\left(\tau ; u-v, \frac{1}{2} \gamma\right) d(\tau v) .
$$

The result (46) follows heuristically, with $c=1$, up to convergence problems in $v$ of the integral.

We now explain how to make this proof rigorous (with $c<1$ ). Given $u, \gamma$, let $\alpha_{0}=\alpha(u, \gamma)$, let $r_{0}=\left(\alpha_{0} / \gamma\right)^{1 / 2}$ and let $\psi_{1}, \psi_{2}$ be $C^{\infty}$ functions with compact support around $p$ in the region $\left|p-p^{\prime}\right|<\left(1-\varepsilon_{1}\right) r_{0}, \varepsilon_{1}>0$ small, and equal to one in the region $\left|p-p^{\prime}\right|<\left(1-\varepsilon_{2}\right) r_{0}$. By writing $1=\psi_{1}+\left(1-\psi_{1}\right)=\psi_{2}+\left(1-\psi_{2}\right), \tilde{f}_{1} \tilde{f}_{2}$ is written as $\left(\tilde{f}_{1} \psi_{1}\right)\left(\tilde{f}_{2} \psi_{2}\right)$ plus other terms whose contributions to $F$ decay exponentially in $\tau$ with rates at least equal (or arbitrarily close) to $\left(1-\varepsilon_{2}\right)^{2} r_{0}^{2} \gamma / 2=$ $\left(1-\varepsilon_{2}\right)^{2} \alpha_{0} / 2$, in view of the vanishing of $1-\psi_{1}$ or $1-\psi_{2}$ in the region $\left|p^{\prime}-p\right|<\left(1-\varepsilon_{2}\right) r_{0}$. The first contribution can be written in a form analogous to (47) except that $F_{1}, F_{2}$ are replaced by the generalized Fourier transforms $\hat{F}_{1}, \hat{F}_{2}$ of $\psi_{1} \tilde{f}_{1}$ and $\psi_{2} \tilde{f}_{2}$ respectively. The rates of exponential fall-off of $\hat{F}_{1}$ and $\hat{F}_{2}$ in $\tau$ are at least equal (or arbitrarily close) to $\operatorname{Inf}\left[\alpha_{1}\left(u, \frac{\gamma}{2}\right),\left(1-\varepsilon_{2}\right)^{2} \alpha_{0} / 2\right]$ and $\operatorname{Inf}\left[\alpha_{2}\left(u, \frac{\gamma}{2}\right),\left(1-\varepsilon_{2}\right)^{2} \alpha_{0} / 2\right]$, so that $\hat{\alpha}_{0}$ is at least equal (or arbitrarily close) to $\left(1-\varepsilon_{2}\right)^{2} \alpha_{0} / 2$. Convergence can now be established along the following lines. For $|v|$ large enough, $u-v \approx-v$ and, in view of the condition (44), $v$-space can be divided into sectors centered around given directions in each of which at least $\alpha_{1}\left(v, \frac{\gamma}{2}\right)$ or $\alpha_{2}\left(-v, \frac{\gamma}{2}\right)$ is $\gtrsim \frac{\alpha_{0}}{2}=\frac{r_{0}^{2} \gamma}{2}$. Since, on the other hand, it is close to $\rho_{1}(\hat{v}, p)^{2} \frac{\gamma}{2}$ $\left(\right.$ or $\left.\rho_{2}(\hat{v}, p)^{2} \frac{\gamma}{2}\right)$ at large $|v|$, i.e. small $\gamma /|v|, \rho_{1}(\hat{v}, p) \gtrsim r_{0}$ (or $\left.\rho_{2}(\hat{v}, p) \gtrsim r_{0}\right)$. In these conditions, results of [9], Appendix 3 ensure that $\hat{F}_{1}$ or $\hat{F}_{2}$ satisfies, around the direction $\hat{v}$ considered, and for large enough $|v|$, uniform bounds including the product of a rapid fall-off factor in $\tau|v|$ and of an exponential fall-off factor $\exp -\left(1-\varepsilon_{2}\right)^{2} r_{0}^{2} \frac{\gamma}{2}$. The desired convergence follows from the rapid fall-off factors, and a uniform rate of exponential fall-off of the integral of the form $\operatorname{cst} \alpha_{0}$ is obtained. Q.E.D.

\section{Appendix 2: Local Momentum-Space Decompositions}

Given any real point $p$, the general mathematical decomposition theorems of $[8,9]$ allow one to derive, from Theorem 5 of Sect. 3, corresponding local momentum space decompositions of $\tilde{T} / \delta\left(\Sigma p_{k}\right)$ or $\widetilde{T}^{\text {amp }} / \delta\left(\Sigma p_{k}\right)$, into sums of boundary values, 
from specified directions, of functions analytic in "local tubes" around p. Such results have first been established in a different way in [5]: see Sect. 1. If one is not interested in precise analyticity domains in complex space but only in the number of terms and in the directions of analyticity, results follow from the microsupport properties (29), as already explained in [6]. More precise results can be established from Theorem 5 as follows. Given $p$, let $r$ be any $>0$ number such that $r<r_{I}^{\text {amp }}(p)$, for some subsets $I$. For simplicity, we consider below the case $r<r_{I}^{\text {amp }}$ for all $I$ such that $r_{I}^{\text {amp }}(p)>0$. Let $F^{\text {amp }}\left(x, p \mid x_{0}\right)$ be the generalized Fourier transform of $\widetilde{T}^{\text {amp }}$ defined by Eq. (1), except that $\gamma|x|$ in the factor $\exp -\gamma|x|\left|p^{\prime}-p\right|^{2}$ is replaced by $x_{0}$, when $x_{0}$ is a supplementary real variable. (No link with time components of the points $x_{i}$.) Theorem 5 entails a bound on $|F|$ including the factor $e^{-r^{2} x_{0}}$ when $\left(x, x_{0}\right)$ lies in the union of the regions $0 \leqq x_{0}<d_{I}(x) / 2 r$ (for all sets $I$ such that $r<r_{I}^{\text {amp }}(p)$ ). This region, at $x_{0}=0$, is the complement of the cone $\Sigma=\bigcap_{I} \Sigma_{I}$, where $\Sigma_{I}$ is the cone in $x$-space defined by the condition $d_{I}(x)=0$. Following [5], $\Sigma$ is contained in the union of (a finite number of) well specified cones $C_{\beta}$ which are closed convex salient cones in $x$-space with apex at the origin modulo global space-time translations. The more detailed analysis then entails bounds on $|F|$ including the factor $e^{-r^{2} x_{0}}$ outside the union of regions $S_{\beta}$ in $\left(x, x_{0}\right)$-space, $x_{0} \geqq 0$, whose traces at $x_{0}=0$ and $x_{0}=1$ are the cones $C_{\beta}$ and sets $C_{\beta}^{(1)}$ respectively. Announced local decompositions of $\widetilde{T}^{\text {amp }} / \delta\left(\Sigma p_{k}^{\prime}\right)$ then follow from results of $[8,9]$. If one e.g. expresses $\widetilde{T}^{\text {amp }} / \delta\left(\Sigma p_{k}^{\prime}\right)$ in terms of the variables $p_{1}^{\prime}, \ldots, p_{N-1}^{\prime}$, one obtains a corresponding decomposition, in the region $\Phi\left(p^{\prime}\right)<r$, where $\Phi\left(p_{1}^{\prime}, \ldots, p_{N-1}^{\prime}\right)=\sum_{k=1}^{N-1}\left|p_{k}^{\prime}-p_{k}\right|^{2}+\left|p_{N}^{\prime}-p_{N}\right|^{2}, p_{N}^{\prime}=-\sum_{k=1}^{N-1} p_{k}^{\prime}$, as a sum of boundary values of analytic functions from the directions of the cones $\Gamma_{\beta}$ dual to the restrictions at $x_{N}=0$ of the cones $C_{\beta}$. These functions are analytic in the local tubes, defined [8] with respect to $\Phi$, whose bases $B_{\beta}$ are the polar sets of the sets $C_{\beta}^{(1)}$ (taken again at $x_{N}=0$ ).

\section{Appendix 3. Macrocausality Properties of Collision Amplitudes - A Simple Example}

We consider below a $2 \rightarrow N-2$ process in which the initial energy-momenta $p_{1}, p_{2}$ are not equal and the initial trajectories $\left(p_{1}, u_{1}\right),\left(p_{2}, u_{2}\right)$ do not meet: see Fig. 3 . This is the type of situation studied in [4] (at $N=2)$, and is one of the situations treated in [7], by different methods (see comments at the end). On the other hand, the analysis of [4], as also to some extent [7], is carried out in a somewhat simplified framework, in comparison with ours. We start here from results of Sect. 2 on $T^{\text {amp }}$ and wish to establish results on connected amplitudes $S^{c}\left(\left\{\hat{\varphi}_{i, \tau}\right\}\right)$.

Whatever $u_{3}, \ldots, u_{N}$ are, $u=\left(u_{1}, \ldots, u_{N}\right)$ is non-causal since the initial trajectories do not meet. Given any $\gamma>0$, a lower $>0$ bound on the rate of exponential decay, depending on the angle $\varphi$ and $\left|u_{1}-u_{2}\right|$, uniform with respect to $p_{3}, \ldots, p_{N}, u_{3}, \ldots, u_{N}$, can be established by introducing e.g., around each initial trajectory, a "security zone" composed of points $v_{i}(i=1,2)$ which either lie at a distance of $u_{i}$ less than $d\left(u_{1}, u_{2}\right) / 3$ or are such that the angle of the direction $\widehat{v_{i}-u_{i}}$ and $p_{i}$ is less than $\rho(\varphi)=\varphi / 3$. The integration domain in (39), i.e. $v$-space, is then divided into (i) the 

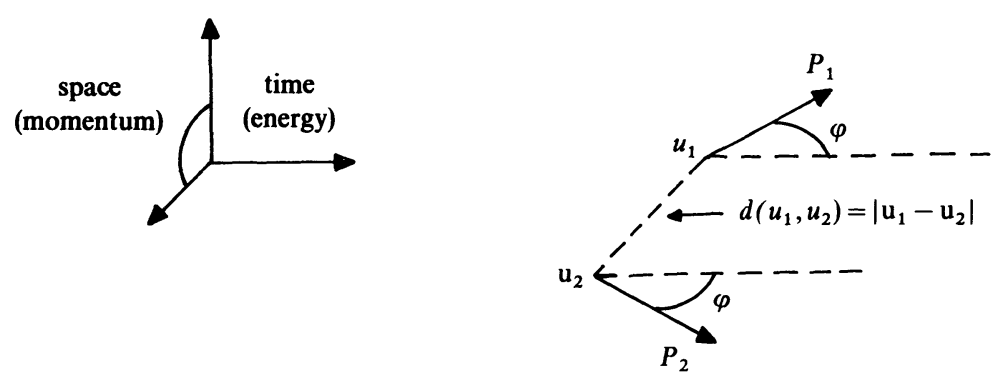

Fig. 3. Two initial trajectories in a $2 \rightarrow N$ process

set of points $v=\left(v_{1}, \ldots, v_{N}\right)$ such that either $v_{1}$ or $v_{2}$ or both lie outside their security zone (ii) its complement (both $v_{1}$ and $v_{2}$ lie inside). In case (i), a minimal rate of exponential fall-off is provided by Theorem 8 (of Sect. 4.1) (with $u$ replaced by $v_{1}-u_{1}$ or $v_{2}-u_{2}$ ). In case (ii), a minimal rate is obtained from results of Sect. 3 relative to $T^{\mathrm{amp}}$. The rate of fall-off obtained for each $v$ is $\operatorname{Sup}_{I} \beta_{I}^{\mathrm{amp}}(p, v ; \gamma)$, where $\beta_{I}^{\mathrm{amp}}$ is defined in Sect. 3. Relevant sets $I$ to be considered are composed of one of the initial indices 1,2 and some (or all) final indices, the Sup being obtained for a set $I$ that depends on $v$.

The minimal rate obtained tends to zero if $\varphi \rightarrow 0$ (existence of points $v$ causal with respect to $T^{\mathrm{amp}}$, in particular $v_{1}=v_{2}$, inducing rates of fall-off of $f_{1, \tau}$ or $f_{2, \tau}$ that tend to zero as $\varphi \rightarrow 0$, since $\rho(\varphi) \rightarrow 0$ ), as also if $\varphi \rightarrow \frac{\pi}{4}$ (high energies) because the relevant set $I$ for some points $v$ is the complement of one initial index, e.g. 1 , and $r_{I}(p)$ which is the distance of $p_{1} \in H_{-}(\mu)$ to $V_{-}(2 \mu)$ tends to zero as $\varphi \rightarrow \frac{\pi}{4}$.

Results are analogous, at least qualitatively, to those obtained in $[4,7]$, which exploit analyticity properties of scattering functions derived from locality and spectrum (as also Lorentz invariance), namely momentum transfer analyticity in the Lehmann ellipse. (As noticed in these works, better results, with a rate of decay that does not tend to zero as $\varphi \rightarrow \frac{\pi}{4}$, can be derived from analyticity in the Martin ellipse whose derivation makes further use of unitarity.)

\section{References}

1. Araki, H., Hepp, K., Ruelle, D.: Helv. Phys. Acta 35, 164 (1962)

2. Haag, R., Swieca, J. A.: Commun. Math. Phys. 1, 308 (1965)

3. Hepp, K.: Axiomatic field theory. Chretien, M., Deser, S. (eds.), p. 137. London: Gordon and Breach 1966

4. Omnes, R.: Phys. Rev. 146, 1123 (1966)

5. Bros, J., Epstein, H., Glaser, V.: Helv. Phys. Acta 45, 149 (1972)

6. Iagolnitzer, D.: The $S$ Matrix, p. 249. Amsterdam: North-Holland 1978, App. 3

7. Manolessou-Grammaticou, M.: Commun. Math. Phys. 46, 223 (1976) 
8. Bros, J., Iagolnitzer, D.: In Proceedings of the 1971 Marseille meeting on Renormalization Theory, and Ann. Inst. H. Poincaré 18 (2), 147 (1973)

9. Iagolnitzer, D.: Introduction to $S$ Matrix Theory, Paris (1972), Chap. II, and Structural Analysis of Collision Amplitudes. Balian, R., Iagolnitzer, D. (eds.), p. 295. Amsterdam: North-Holland 1976

10. Bony, J. M.: Séminaire Goulaouic-Schwartz, Ecole Polytechnique, 1976-77, exposé n ${ }^{\circ} 3$

11. Hörmander, L.: Acta Math. 127, 79 (1971)

12. Sato, M., Kawai, T., Kashiwara, M.: Hyperfunctions and pseudo-differential equations. Lecture Notes in Mathematics, Part III. Berlin, Heidelberg, New York: Springer 1973

13. Iagolnitzer, D., Stapp, H. P.: Commun. Math. Phys. 14, 15 (1969)

14. Bogoliubov, N. N., Logunov, A. A., Todorov, I. T.: Introduction to Axiomatic Field Theory. Reading, MA: Benjamin W. A. 1975, p. 359 and references therein to original works

Communicated by K. Gawedzki 
